DOI: $10.24850 / \mathrm{j}$-tyca-2019-06-D1

Discusión

\title{
Discusión a la nota técnica "Índices de cambio climático en la cuenca del Río Grande, Chiapas, México" de José Alonso Figueroa-Gallegos, 8(6), noviembre-diciembre 2017, 137-143
}

Martín D. Mundo-Molina ${ }^{1}$

${ }^{1}$ Centro de Investigación de la Facultad de Ingeniería, Universidad Autónoma de Chiapas, Chiapas, México, ic_ingenieros@yahoo.com.mx

Recibido: $12 / 03 / 2019$

Aceptado: 13/03/2019

\section{Cambio climático: IPCC 2007 Y CMNUCC 2014}

El autor, Figueroa-Gallegos (2017), escribió una nota técnica en la cual estudia los índices de cambio climático en la cuenca RD30Gl del Río Grande Comitán en el estado de Chiapas. Desde el epígrafe surge la deliberación académica sobre esta nota técnica. La cuenca del Río Grande Comitán no es una cuenca en la terminología hidrográfica como el título lo refiere, sino una subcuenca ubicada en la región hidrológica RH 30 Grijalva-Usumacinta, en la subregión 30C Usumacinta, insertada en la subcuenca del río Lacantún. El estudio que el autor ejecutó en la subcuenca RD30Gl Río Grande está enmarcado en el calentamiento global, desde la perspectiva del cambio climático, basado en una definición no vigente del IPCC (2007) que cita de forma literal en la introducción de su trabajo: "...la variación del estado del clima, que puede deberse a procesos internos naturales, a forzamientos externos o a cambios antropogénicos persistentes de la composición de la atmósfera". La definición de cambio climático vigente la actualizó la Convención Marco de las Naciones Unidas sobre el Cambio Climático (CMNUCC) en el año 2014: "... es el cambio de clima atribuido directa o indirectamente a la actividad humana que altera la composición de la atmósfera global que se suma a la variabilidad natural del clima observada durante períodos de tiempo comparables (IPCC, 2014)". Ésta 
elimina los forzamientos externos enunciados por el IPPC (2007) e introduce tres elementos notables: 1. En primer lugar enuncia que el cambio de clima puede ser atribuido directa o indirectamente a la actividad humana; 2 . Que puede alterar la composición de la atmósfera global, y 3. Confirma que las comparaciones de la variabilidad climática natural observada deben realizarse en periodos comparables. Como se puede apreciar, la fuente del IPCC (2007) citada por el autor no es la más reciente, en una rama de la ciencia en donde de forma periódica y permanente esta institución reporta y actualiza el estado de los avances en los diferentes campos de la misma.

\section{Diversidad geomeorfológica y climática de la subcuenca RD30GI}

Para analizar la variabilidad climática en toda la subcuenca a través de los índices climáticos del ETCCDI, el autor utilizó únicamente tres estaciones meteorológicas estándar en una superficie de $6212.51 \mathrm{~km}^{2}$, afirmando en el resumen que las tres están ubicadas dentro de la subcuenca. Sin embargo, se puede probar que sólo una se ubica dentro de la misma. Además, la que se ubica dentro de la subcuenca, la estación 07205 Comitán, se localiza en la parte más septentrional (figura 1), en la unidad morfológica $\mathrm{V}$ (terraza estructural) descrita por Mora, Bonifaz y López (2016), cuyas variables meteorológicas sólo tienen un alcance que no va más allá de una pequeña parte del área donde está situada, porque está inserta en la ciudad de Comitán, donde mide las temperaturas de las islas de calor generadas por el pavimento, las estructuras de concreto y ladrillo de las viviendas que la rodean. La estación meteorológica no cumple con ninguna de las recomendaciones de instalación sugeridas en Doorenbos (1976) por su inadecuada ubicación.

Con una sola estación y además ubicada dentro de la ciudad, no se pueden caracterizar los índices de cambio climático en la subcuenca del Río Grande como el título del trabajo lo indica, porque justamente la subcuenca se singulariza por tener diversos tipos de climas. Una parte del desencuentro entre el título del trabajo y las conclusiones se debe a que el autor no tomó en cuenta la relación morfométrica-hidrológica de 
la subcuenca, ésta es de clase III y de acuerdo con la relaciones matemáticas mostradas en Campos (1998), González (2004), y Martínez y Díaz (2011) tiene un índice de compacidad mayor a dos, que tipifica a una subcuenca oblonga (figura1), cuyos gradientes altitudinales generan diversos tipos de climas, de extremo a extremo, en su parte más alargada.

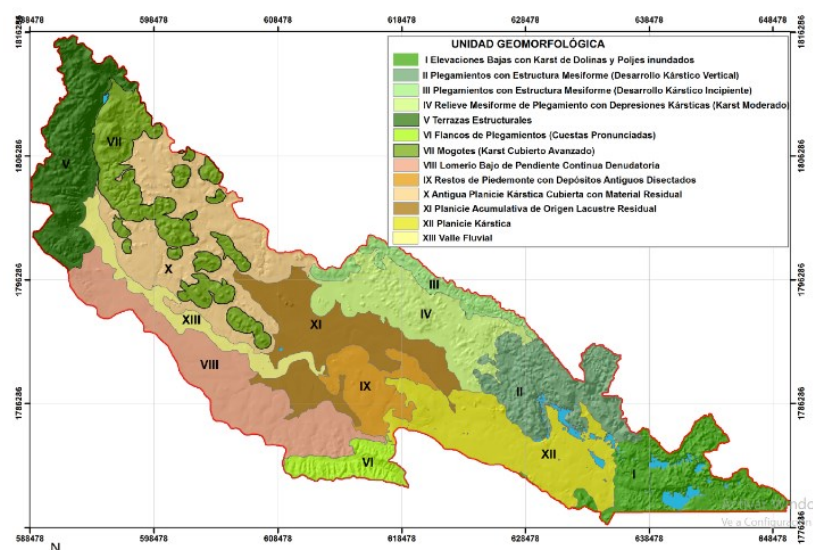

Figura 1. Unidades geomorfológicas de la subcuenca del Río Grande (Mora et al., 2016). En rojo se muestra la ubicación de la estación 07205.

De acuerdo con Mora et al. (2016), la subcuenca mostrada en la figura 1 es kárstica, conformada por secuencias sedimentarias de génesis marino y transicional. Así, la gran diversidad de climas de la subcuenca se debe al origen paleoestructural de su forma que ha sido determinado a su vez por una amplia variedad de relaciones endógenas edáficas, topográficas, biológicas y climáticas, constituida al menos por trece unidades geomorfológicas agrupadas en configuraciones de montaña (seis unidades, con pendientes de hasta $55.5^{\circ}$ ); lomerío (tres), y planicie (cuatro), donde la evolución de las relaciones endógenas arriba citadas depende de las interacciones: roca-agua, condiciones climáticas, topografía y la acción biológica de cada unidad (Ford \& Williams, 1989; Ford \& Williams, 2007; Stokes, Griffiths, \& Ramsey, 2010). Cada unidad tiene un clima dominante con diversos microclimas en función de la rugosidad y la pendiente de cada una de ellas (Martínez \& Senciales, 2003). De esta manera, la expresión geodinámica (orografía) y biológica diversa de la subcuenca permite generar diversos tipos de clima que posibilita la existencia de diferentes clases de bosques (coníferas, 
mesófilos de montaña y encinos), selva perennifolia, selva caducifolia, selvas bajas y vegetación inducida como se refiere en GEC (2013) y Mundo-Molina (2017). Por lo tanto, los estudios que sobre variabilidad climática se realicen en la subcuenca, basados en estaciones terrestres, sólo serán válidos para las pequeñas áreas de influencia de cada estación, pero no para toda la subcuenca.

\section{Tendencias de calentamiento y escalas meteorológicas}

Por otra parte, sobre los resultados encontrados en la estación 07205 Comitán y 07104 Margaritas, el autor afirmó en el resumen y en las conclusiones de su trabajo: "...se puede concluir que la temperatura mínima y máxima presenta una tendencia ascendente significativa, que muestra coincidencia con lo reportado por Campos-Aranda (2015) para el estado de Zacatecas, y con las tendencias previstas para el estado de Chiapas reportada por Ramos (2010)". Por un lado, las tendencias de calentamiento mostradas por el autor en las estaciones antes enunciadas tienen una inadecuada interpretación de los resultados, como se mostrará más adelante, y por otro, si se atiende al título, la tendencia subrayada avala que dicho calentamiento se está generando en toda la subcuenca RD30Gl Río Grande, porque apoya sus resultados en los argumentos de incremento de temperatura señaladas por Ramos (2010) en las simulaciones realizadas con el modelo japonés TL959 para todo el estado de Chiapas; si el autor arguye que las tendencias de calentamiento encontradas en las estaciones 07205 Comitán y 07104 Margaritas sólo tienen validez paras las pequeñas áreas geográficas de donde proceden, entonces no debió comparar y/o apoyar sus resultados locales con lo reportado por Ramos (2010), porque las escalas meteorológicas no son las mismas (una son locales y la otra es sinóptica); amén de que deberá revisarse como sumo cuidado, como lo indica el IPCC (2014), si los periodos de tiempo usados son comparables. 


\section{Ciclos de calentamiento-enfriamiento de la estación 07205}

Los objetivos, resultados y conclusiones de la nota técnica no se demostraron y son discordantes. El autor afirma en el apartado de materiales y métodos que su objetivo fue contribuir al conocimiento de la variabilidad climática en el marco del cambio global, analizando la evolución de las precipitaciones y temperaturas que permiten explicar los posibles patrones de cambio a través de los índices climáticos del ETCCDI. Sin embargo, no analizó las precipitaciones y no presentó sus conclusiones sobre éstas. Respecto a las temperaturas su conclusión más importante enuncia: "...las temperaturas mínima y máxima presentan una tendencia ascendente significativa para las estaciones Comitán y Margaritas" (se hace notar que la temperatura mínima como tal no está enunciada en el cuadro 3 de su nota técnica). Así, el polemista confirma que no se percibe una contribución del autor en el conocimiento de la variabilidad climática en la subcuenca, puesto que no explica la evolución de las temperaturas en los más de 50 años de registros diarios de ambas estaciones meteorológicas, ni sus patrones de cambio. Es decir, sólo se limita a enunciar las tendencias de la estación Comitán y Margaritas, amén de que dichas tendencias debieron analizarse con un método estadístico para aceptar o rechazar la hipótesis nula $H_{0}$ con un nivel de significancia de 2.5 o 5\%, como se muestra en Cantor (2011), o Martínez-Austria, Patiño-Gómez y Tamayo (2014), y haber explicado, como se indica en los objetivos, las justificaciones de la tendencia de incremento encontradas; en otras palabras, si son atribuibles a la actividad humana o a la variabilidad natural del clima.

Por otra parte, no existe tendencia de incremento de la temperatura máxima en la estación meteorológica 07205 Comitán, como afirma el autor. El polemista estudió con detalle la evolución de los procesos cíclicos de enfriamiento-calentamiento de 54 años de registros diarios de temperatura y sus anomalías de la estación 07205, y demostró que efectivamente, existen más ciclos de calentamiento que de enfriamiento, pero sólo en las temperaturas medias y mínimas, no en las temperaturas máximas (Mundo-Molina, 2017). La diferencia de estos resultados deben buscarse por tres rutas paralelas: en el relleno de datos faltantes (se sugiere usar el método WS); en el análisis de la 
homogeneidad de la serie temporal originalmente inhomogénea (figura 2 ), la cual se debe verificar al menos con tres técnicas distintas (SNHT, Von Newman, Buishand); y el método de homogenización de la serie mostrada en la figura de abajo, que debe ejecutarse usando climatol, para ser congruentes con los métodos estadísticos usados en el estados actual de las investigaciones en este rubro.

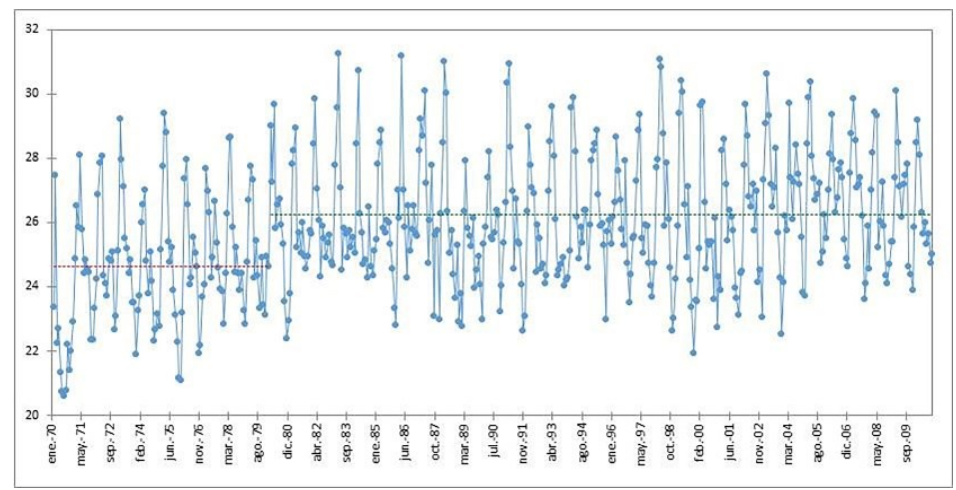

Figura 2. Temperaturas no homogéneas, estación 07205 (MundoMolina, 2017).

\section{Sobre la interpretación de los resultados y las conclusiones}

Cuando en el campo de las ciencias se utilizan argumentos que "suponen y afirman algo" que no se conoce con certeza científica, o no se fundamenta y demuestra con bases teóricas o experimentales sólidas, se generan especulaciones. Bajo estas circunstancias se aprecian tres especulaciones en las conclusiones del autor:

1. "...las temperaturas mínima y máxima presentan una tendencia ascendente significativa".

2. "...es necesario realizar este tipo de análisis en otras cuencas, a fin de poder establecer si las tendencias aquí detectadas son generalizadas $y$ se puedan establecer medidas orientadas a redimensionar los sistemas de abastecimiento de todo tipo, pues 
la evaporación sin duda estará aumentando, disminuyendo la disponibilidad de agua e incrementando las necesidades".

3. "...se puede concluir, [que] las temperaturas mínima y máxima presentan una tendencia ascendente significativa, que muestra coincidencia con lo reportado por Campos-Aranda (2015) para el estado de Zacatecas, y con las tendencias previstas para el estado de Chiapas reportadas por Ramos (2010)".

En el primer caso: el autor no consideró las circunstancias reales que rodean a las estaciones meteorológicas 07205 y 07104 . Ambas muestran tendencias positivas de calentamiento porque están insertadas dentro de las ciudades de Comitán y Las Margaritas, respectivamente, ubicadas cerca de casas habitación, donde se generan islas de calor, producto de la radiación solar reflejada de los tejados, techos de concreto, paredes de ladrillo, pavimentos y obras civiles; se suma a estas circunstancias el cambio de uso de suelo y la deforestación en torno al medio ambiente que las rodea (Mundo-Molina, 2017), por lo tanto generan una tendencia de incremento de temperatura como lo demuestra el análisis estadístico. Sin embargo, esta tendencia de incremento de temperatura no se repite en la estación "La Soledad" (07062), localizada a $33 \mathrm{~km}$ de la estación Comitán y a $15 \mathrm{~km}$ de la estación Margaritas (en línea recta). La estación La Soledad no tiene tendencias de calentamiento porque se ubica en una zona agrícola, a las afueras del ejido Chiapas, sin casas que la circunden ni zonas asfaltadas y la deforestación es mínima comparada a las dos estaciones antes referidas. "La Soledad" refleja de forma más fiel la climatología natural de su zona de influencia, sin islas de calor urbano.

En el segundo caso: el autor afirma, apoyado en la primera conclusión que: "... la evaporación sin duda estará aumentando, disminuyendo la disponibilidad de agua e incrementando las necesidades", sin embargo no presenta pruebas de ello.

En el tercer caso: no se deben comparar los resultados de las tendencias de variación climática obtenidas con estaciones meteorológicas terrestres, respecto a las simuladas con modelos de circulación general de la atmósfera (MCGA), porque la primera tiene alcances locales y algunas veces regionales (dependiendo de la densidad territorial de las estaciones meteorológicas), mientras que en la segunda su alcance espacial es sinóptico; por esa razón, los MCGA no son capaces aún de simular adecuadamente las variaciones climáticas a nivel local o regional, por problemas justamente de escala espacial; en consecuencia, las unidades de estudio de cuencas hidrológicas 
completas con sus bosques, regiones agrícolas y condiciones topográficas no son definidas con exactitud en el mallado de los modelos.

Aunque muchos MCGA permiten modelar matemáticamente, la CGA terrestre, el hielo o los océanos, usando las ecuaciones de Navier-Stokes en coordenadas polares de forma acoplada o no acoplada, para obtener evidencias científicas de los posibles impactos del calentamiento global (Palacios \& Serrano, 2011), no todos los modelos son aplicables a todas la regiones del mundo. En este marco, los resultados que el autor tomó de Ramos (2010) afirman que las tendencias climatológicas previstas en el estado de Chiapas se relacionan con el incremento de la temperatura media proyectada bajo el escenario SRES A1B, con un incremento en la temperatura máxima de entre 3 y $3.6^{\circ} \mathrm{C}$ y para la temperatura mínima entre 2.5 y $2.8^{\circ} \mathrm{C}$, respectivamente; estos resultados se obtuvieron con el MCGA denominado modelo japonés TL959. Debe notarse en primera instancia que esta afirmación se generalizó para todo el estado de Chiapas, sin considerar la relevancia de los gradientes topográficos abruptos de la topografía chiapaneca. La topografía tiene mucha relevancia en los MCGA, como lo confirmaron Palacios y Serrano (2011), quienes aseveraron que a pesar de que el modelo japonés TL959 es un modelo de alta resolución espacial y es capaz de recrear la circulación atmosférica general en mallados de $20 \times 20 \mathrm{~km}$, encontraron que es necesario mejorar la parametrización física del TL959 en zonas motañosas de gran altura, ya que en estas regiones se encontró el mayor desajuste del modelo cuando se intentó validar sobre la climatología de la República de Ecuador en las variables de precipitación y temperaturas extremas.

Por otro lado, el Cuarto Informe de Evaluación (AR4) del Panel Intergubernamental de Cambio Climático recomendó 21 MCGA potencialmente utilizables a escala mundial para obtener evidencias científicas de los posibles impactos del calentamiento global (Mora, Liu, Cisneros, Wyseure, \& Willems, 2012), pero como ya se ha indicado no todos son útiles para México. Uno de ellos es el modelo japonés TL959, que ya no fue incluido en el Quinto Informe; si bien tiene una resolución más alta que el resto de los modelos, no trabaja de forma acoplada con la capa de hielo o de los océanos. De este conjunto de argumentos se desprende que los resultados del modelo japonés TL959 para el caso de México tiene mucha incertidumbre. Para el estado de Chiapas sus resultados son aún más dudosos. 


\section{Referencias}

Campos, A. F. (1998). Procesos del ciclo hidrológico. San Luis Potosí, México: Universidad Autónoma de San Luis Potosí.

Campos-Aranda, D. (2015). Búsqueda del cambio climático en la temperatura máxima de mayo en 16 estaciones climatológicas del estado de Zacatecas, México. Tecnología y ciencias del agua, 6(3), 143-160.

Cantor, G. D. (2011). Evaluación y análisis espaciotemporal de tendencias de largo plazo en la hidroclimatología colombiana. Bogotá, Colombia: Universidad Nacional de Colombia.

Doorenbos, J. (1976). Estaciones agrometeorológicas. Roma, Italia: Organización de las Naciones Unidas para la Agricultura y la Alimentación, FAO.

Figueroa-Gallegos, J. A. (2017). Índices de cambio climático en la cuenca del Río Grande, Chiapas, México. Tecnología y ciencias del agua, 8(6), 137-143.

Ford, D. C., \& Williams, P. W. (1989). Karst Geomorphology and Hydrology: Unwin Hyman. London, UK: John Wiley \& Sons Ltd.

Ford, D. C., Williams, P. W. (2007). Karst hydrogeology and geomorphology. Chichester, UK: John Wiley \& Sons, Ltd.

GEC, Gobierno del Estado de Chiapas. (2013). Programa regional de desarrollo 2013-2018. Región XV Meseta Tojolabal Comiteca. Tuxtla Gutiérrez, México: Gobierno del Estado de Chiapas.

González, A. I. (2004). Análisis morfométrico de la cuenca y de la red de drenaje del río Zadorra y sus afluentes aplicado a la peligrosidad de crecidas. Boletín de la AGE, Geografía, Prehistoria y Arqueología, Universidad del País Vasco, (38), 311-329.

IPCC, Grupo Intergubernamental de Expertos sobre el Cambio Climático. (2014). Cambio climático 2014: informe de síntesis. Contribución de los Grupos de trabajo I, II y III al Quinto Informe de Evaluación del Grupo Intergubernamental de Expertos sobre el Cambio Climático. Ginebra, Suiza: Grupo Intergubernamental de Expertos sobre el Cambio Climático.

IPCC, Grupo Intergubernamental de Expertos sobre el Cambio Climático. (2007). Climate Change 2007- The Physical Science Basis. Cambridge, UK: Cambridge University Press.

Martínez, M. J., \& Senciales, M. J. (2003). Morfogénesis y procesos edáficos. El caso de los montes de Málga. Baética, 25, 219-257. 
Martínez, G., \& Díaz, J. (2011). Morfometría en la cuenca hidrológica de San José del Cabo, Baja California Sur, México. Revista Geológica de América Central, 44, 83-100.

Martínez-Austria, P. F., Patiño-Gómez, C., \& Tamayo, J. E. (2014). Análisis de tendencias climáticas en la cuenca baja del río Yaqui, Sonora, México. Tecnología y ciencias del agua, 5(6), 135-149.

Mora, D. E., Liu, T., Cisneros, F., Wyseure, G., \& Willems, P. (2012). Statistical analysis on the performance of global and regional climate models for the paute river basin in the south-ecuadorian Andes. 10th International Conference on Hydroinformatics, Hamburg, Germany.

Mora, P. L., Bonifaz, R., \& López, M. R. (2016). Unidades geomorfológicas de la cuenca del Río Grande de Comitán, Lagos de Montebello. Boletín de la Sociedad Geológica Mexicana, 68(3), 377-394.

Mundo-Molina, M. (2017). Análisis de los procesos cíclicos de enfriamiento-calentamiento y anomalías de las temperaturas máximas y mínimas de la estación meteorológica 07205 ubicada en la meseta comiteca de la RH Grijalva Usumacinta, en el marco de la inestabilidad climática global. En: Soares, D., \& García, G. A. (2017). La cuenca del río Usumacinta, desde la perspectiva del cambio climático. Jiutepec, México: Instituto Mexicano de Tecnología del Agua.

Palacios, E., \& Serrano, S. (2011). Validación de los modelos de cambio climático hidrostáticos y no hidrostáticos sobre la climatología del Ecuador en las variables de precipitación y temperaturas extremas. La Granja, 13(1), 21-30.

Ramos, S. (2010). Escenarios climáticos para el estado de Chiapas. Informe final. Fase II. Tuxtla Gutiérrez, México: Universidad de Ciencias y Artes de Chiapas, UNICACH.

Stokes, T., Griffiths, P., \& Ramsey, C. (2010). Compendium of forest hydrology and geomorphology in British Columbia. In: Pike, R. G., Redding, T. E., Moore, R. D., Winker, R. D., \& Bladon, K.D. (eds.). Karst geomorphology, hydrology, and management. Recuperado de http://www.for.gov. bc.ca/hfd/pubs/Docs/Lmh/Lmh66.htm 